\title{
PROPOSTA DE PROTOCOLO DE AVALIAÇÃO CLÍNICA DA FUNÇÃO MASTIGATÓRIA
}

\section{Proposal for mastication clinical evaluation protocol}

\author{
Melina Evangelista Whitaker ${ }^{(1)}$, Alceu Sérgio Trindade Júnior ${ }^{(2)}$, Katia Flores Genaro ${ }^{(3)}$
}

\begin{abstract}
RESUMO
Objetivo: elaborar um protocolo de avaliação clínica da função mastigatória, visando auxiliar o fonoaudiólogo a identificar e interpretar os sinais clínicos de alterações na dinâmica da mastigação, além de possibilitar comparações de acompanhamento de tratamento e entre diferentes avaliadores. Métodos: este protocolo foi elaborado com base na prática clínica de fonoaudiólogos que responderam a um questionário enviado por e-mail; na literatura, segundo a identificação dos pontos comuns a todos os protocolos de avaliação da mastigação; além da experiência dos fonoaudiólogos do Hospital de Reabilitação de Anomalias Craniofaciais da Universidade de São Paulo. Resultados: o protocolo de avaliação clínica da mastigação contempla aspectos relacionados aos aspectos morfológicos e à neurofisiologia da função mastigatória: Conclusão: este trabalho contribui com a Fonoaudiologia, uma vez que busca, de maneira mais completa possível, critérios para a avaliação da mastigação, norteando a atuação fonoaudiológica clínica baseada em evidências.
\end{abstract}

DESCRITORES: Avaliação; Mastigação; Sistema Estomatognático

\section{INTRODUÇÃO}

O fonoaudiólogo que atua na área de Motricidade Orofacial volta seu trabalho para a pesquisa, prevenção, avaliação, diagnóstico, desenvolvimento funcional, habilitação, aperfeiçoamento e reabilitação dos aspectos estruturais e funcionais das regiões orofacial e cervical ${ }^{1}$.

Dentre as funções orais tem-se a mastigação e, portanto, o fonoaudiólogo é um dos profissionais aptos a diagnosticar as disfunções mastigatórias, bem como tratá-las. Ressalta-se que para que o trabalho seja realizado adequadamente, faz-se necessário o diagnóstico preciso e correto da alteração, envolvendo a avaliação detalhada da função

(1) Fonoaudióloga do Hospital de Reabilitação de Anomalias Craniofaciais da Universidade de São Paulo, HRAC-USP, Bauru, SP; Mestre em Ciências da Reabilitação pelo Hospital Reabilitação de Anomalias Craniofaciais da Universidade de São Paulo.

(2) Cirurgião - Dentista; Docente do Departamento de Ciências Biológicas da Faculdade de Odontologia de Bauru da Universidade de São Paulo, FOB-USP, Bauru, SP; Professor Titular.

(3) Fonoaudióloga; Docente do Departamento de Fonoaudiologia da Faculdade de Odontologia de Bauru da Universidade de São Paulo, FOB-USP, Bauru, SP; Livre-Docente.

Conflito de interesses: inexistente envolvida, assim como das demais funções relacionadas ${ }^{2-5}$. Frente à dificuldade de se encontrar instrumentos clínicos padronizados para tal, surgiu a proposta deste trabalho.

A mastigação é o ato de morder e triturar o alimento, a qual constitui um ato fisiológico e complexo, que envolve atividades neuromusculares e digestivas. Com relação a esta última função, destaca-se a ação da amilase salivar durante a trituração dos alimentos, facilitando a deglutição e a ação das enzimas digestivas do estômago e, principalmente, do pâncreas. O sistema mastigatório pode ser considerado como uma unidade funcional constituída pelos seguintes componentes: dentição, estruturas periodontais de suporte maxilar e mandibular, articulação temporomandibular, musculatura mastigatória e de lábios, bochechas e língua, tecidos moles que revestem essas estruturas, assim como a inervação e vascularização que suprem esses componentes ${ }^{2,3}$. É uma função aprendida que depende de vias neurais e conexões sinápticas estabelecidas e comandadas pelo córtex cerebral. Assim, aparece posteriormente devido ao aumento do espaço intra-oral, a irrupção dos dentes, a maturação de todo arcabouço neuromuscular e o processo, em curso, de remodelação das articulações tempomandibulares ${ }^{4-6}$. 
Existem vários fatores que influenciam a função mastigatória de forma a alterá-la. Esses fatores podem envolver alterações estruturais ou funcionais do sistema estomatognático, que desencadeiam um desequilíbrio, do qual um dos sinais pode ser a disfunção mastigatória. Assim, devido à complexidade da função mastigatória, são realizadas análises de cada aspecto da mastigação, como duração dos atos e ciclos mastigatórios, força de mordida, movimentos mandibulares, eficiência mastigatória, entre outros, com técnicas diferenciadas tecnologicamente. Desta forma, o diagnóstico das alterações mastigatórias, e das estruturas que a envolvem, torna-se preciso e o tratamento direcionado para cada caso. Alguns métodos instrumentais de avaliação possibilitam a obtenção de dados objetivos, porém, nem sempre fazem parte da prática clínica fonoaudiológica. Deste modo, torna-se necessário que a avaliação clínica da mastigação, ou seja, não instrumental, possa analisar individualmente cada aspecto e, ao mesmo tempo, avaliar a função mastigatória como um todo, de forma prática e padronizada.

Nesse sentido, a literatura é muito escassa no que diz respeito aos métodos não instrumentais padronizados para o estudo da mastigação, uma vez que as pesquisas priorizam os métodos instrumentais, em detrimento dos não instrumentais. Isto prejudica a objetividade dos dados, tanto em pesquisa quanto na comparação de casos clínicos, quando a avaliação é realizada por avaliadores distintos sem o mesmo treinamento ou formação.

Assim, o objetivo deste trabalho foi elaborar um protocolo de avaliação clínica da função mastigatória, visando auxiliar o fonoaudiólogo a identificar e interpretar os sinais clínicos de alterações na dinâmica da mastigação, além de possibilitar comparações de acompanhamento de tratamento e entre diferentes avaliadores.

\section{MÉTODOS}

Elaborou-se um questionário (Figura 1) a ser respondido por fonoaudiólogos de diferentes regiões do Brasil, a fim de se obter dados sobre a avaliação da função mastigatória realizada pelos mesmos. Esse questionário constava de 5 questões envolvendo dados da história clínica, aspectos morfológicos do sistema estomatognático, assim como a avaliação da função mastigatória propriamente dita. Essas questões eram descritivas e o fonoaudiólogo deveria relatar os aspectos observados, os materiais e procedimentos utilizados, os exames complementares realizados, entre outros; bem como deveria citar se outras funções orais eram investi- gadas, descrevendo quais funções, como avaliava e a justificativa para a verificação dessa função.

Os questionários foram enviados por meio de correio eletrônico e, ao reenviar o questionário preenchido ao e-mail da autora, os fonoaudiólogos participantes, firmavam seu Consentimento Livre e Esclarecido.

De um total de 350 questionários enviados, 70 retornaram preenchidos. Após análise dos questionários recebidos e da literatura consultada, um protocolo de avaliação foi proposto.

Este projeto de pesquisa recebeu aprovação do Comitê de Ética em Pesquisa da Instituição onde o mesmo foi realizado (protocolo 288/2003).

\section{RESULTADOS}

Para facilitar a análise da avaliação da mastigação, além da observação feita pelo profissional durante o processo de avaliação, também se recomenda a filmagem desse procedimento para todos os casos, a fim de se realizar uma análise mais detalhada posteriormente. Propõe-se que a filmadora esteja posicionada em tripé, sempre a mesma distância da cadeira do paciente, que deverá estar sentado com apoio plantar adequado e em uma cadeira sem apoio para a cabeça, de modo a não limitar seus movimentos. Um painel que trace o plano horizontal e vertical deve ser posicionado atrás dele, servindo de referência em relação à postura corporal e dos órgãos que participam da mastigação ${ }^{7}$. Ressalta-se que este método possui vantagens como ser um método acessível, de baixo custo, evitando-se a repetição da avaliação funcional por várias vezes e possibilitando uma análise sem a presença do paciente. Como desvantagens, pode-se destacar a necessidade de critérios para a mesma, visando à comparação entre si, como a padronização da mesma distância entre a filmadora e o paciente, a luminosidade adequada da sala de filmagem, a marcação dos planos horizontal e vertical atrás do paciente, além de possibilidade de constranger o paciente durante a avaliação, modificando sua mastigação habitual na presença da câmera.

A seguir encontram-se os itens propostos para a avaliação e a descrição da forma de avaliação dos mesmos e, em anexo é apresentada a ficha de avaliação elaborada. Na mesma constam: a identificação do paciente, incluindo seus dados pessoais, escolaridade, profissão, endereço; além da identificação do profissional que está solicitando a avaliação. 
HosPitAl de REABILITAČÃO

DE ANOMALIAS CRANIOFACIAIS UNIVERSIDADE DE SÁO PAULO

\section{Avaliação Clínica da Função Mastigatória}

\section{IDENTIFICAÇÃO}

Nome:

Gênero:

$\begin{array}{ll}\mathrm{D} / \mathrm{N}: & \text { Idade: } \\ \text { Escolaridade: } & \text { Profissão: }\end{array}$

Telefone:

Encaminhado por:

Endereço: Cidade/Estado:

\section{FUNÇÃO MASTIGATÓRIA}

1. Alimentos testados:

2. Tamanho da porção :

3. Apreensão: $\square$ anterior

$$
\begin{aligned}
& \square \text { lateral } \\
& \square \text { posterior }
\end{aligned}
$$

4. Movimento mandibular: $\square$ vertical e lateral

$\square$ parte com as mãos

$\square$ vertical

$\square$ mínimo

$\square$ quebra com os dentes

5. Lado inicial da função: Teste 1: $\square D \square E$ Teste 2: $\square D \square E$

Teste 3: $\square D \square$

$\square$ ausente

Teste 4: $\square D \square E$

6. Tipo mastigatório: $\square$ bilateral al ternado

$\square$ bilateral simultâneo

$\square$ unilat. preferencial

$\square$ unilat. crônico

7. Musculatura perioral: $\square$ ausente

$$
\begin{aligned}
& \square \text { pouca } \\
& \square \text { acentuada }
\end{aligned}
$$

8. Lábios: $\square$ selados

$\square$ af astados as vezes

$\square$ afastados

9. Tremor: $\square$ ausente

$$
\begin{aligned}
& \square \text { lábios } \\
& \square \text { língua } \\
& \square \text { mandíbula }
\end{aligned}
$$

10. Contração de masseteres: $\square$ forte

$$
\begin{aligned}
& \square \text { regular } \\
& \square \text { fraca }
\end{aligned}
$$

11. Coordenação dos movimentos: Dadequada

$$
\square \text { inadequada }
$$

13. Amassamento com a língua: $\square$ ausente

$$
\square \text { presente }
$$

15. Local da trituração: $\square$ anterior

$$
\square \text { posterior }
$$

17. Ruídos nas ATMs: $\square$ ausente

$$
\begin{aligned}
& \square \text { estalo } \\
& \square \text { crepitação }
\end{aligned}
$$

12. Escape anterior do alimento: $\square$ ausente

$$
\square \text { presente }
$$

14. Movimento de cabeça: $\square$ ausente

$$
\square \text { presente }
$$

16. Respiração durante a função: $\square$ nasal

$$
\square \text { oro-nasal/oral }
$$

18. Tempo mastigatório ( seg.)

$$
\begin{aligned}
& \square \text { adequado } \\
& \square \text { lento } \\
& \square \text { rápido }
\end{aligned}
$$

19. N. ${ }^{\circ}$ de ciclos mastigatórios:

20. Formação do bolo: $\square$ completo

$$
\begin{aligned}
& \square \text { parcial } \\
& \square \text { não forma }
\end{aligned}
$$

$$
\begin{aligned}
& \text { 21. Presença de: } \begin{aligned}
\square \text { tosse } \\
\\
\square \text { engasgo } \\
\\
\square \text { dispnéia } \\
\square \text { alteração na coloração facial }
\end{aligned}
\end{aligned}
$$


23. Teste de eficiência mastigatória:

Amêndoa 1 (mastigação habitual): Tempo: ___ segundos

N. ${ }^{\circ}$ de ciclos:

Tipo de partículas: $\square A \quad \square B \quad \square C \quad \square D \quad \square E$

Amêndoa 2 (10 segundos):

Tipo de partículas: $\square A \quad \square B \quad \square C \quad \square D \quad \square E$

Amêndoa 3 (20 segundos):

Tipo de partículas: $\square A \quad \square B \quad \square C \quad \square D \quad \square E$

Amêndoa 4 (40 segundos):

Tipo de partículas: $\square A \quad \square B \quad \square C \quad \square D \square E$

Amêndoa 5 (tempo e $n .^{\circ}$ de ciclos da amêndoa 1).

Tipo de partículas: $\square A \quad \square B \quad \square C \quad \square D \quad \square E$

Classificação: $\quad$ EM ótima (partículas B na $2^{a}$ Amêndoa)

- EM boa (partículas $B$ na $3^{a}$ Amêndoa)

- EM regular (partículas $A$ e $B$ na $3^{a}$ Amêndoa)

- EM ruim (ausência de partículas $A$ na $4^{a}$ Amêndoa)

$\square$ EM péssima (presença de partículas $A$ na $4^{a}$ Amêndoa)

\section{SÍNTESE DO CASO}

\section{CONDUTA}

Encaminhamento (área/motivo):

$\square$ Terapia miofuncional:

Avaliador:

Data:

Figura 1 - Protocolo utilizado (continuação)

Rev CEFAC, v.11, Supl3, 311-323, 2009 


\section{Alimentos testados}

Sugere-se que a avaliação da mastigação seja realizada com pão ou biscoito tipo wafer, sendo o primeiro de custo baixo e de fácil aceitação entre os pacientes e o segundo apresenta um tempo maior de conservação, sem modificação de suas propriedades. Além disso, existem parâmetros em relação ao tempo mastigatório, número e duração dos ciclos mastigatórios ${ }^{8,9}$. Para a avaliação da eficiência mastigatória, recomenda-se a utilização de amêndoas ${ }^{10}$

\section{Tamanho do alimento}

Sugere-se uma porção de $2 \mathrm{~cm}$ ou $1 / 4$ de biscoito tipo waffer e, quando for utilizado o pão francês, recomenda-se o mini pão francês, cortando-se uma fatia da região central do pão, de $1 \mathrm{~cm}$ de espessura e tomando-se $1 / 4$ desta fatia ${ }^{8,9}$. Para a avaliação da eficiência mastigatória são necessárias 5 amêndoas, a serem consumidas uma por vez ${ }^{10}$.

\section{Preensão do alimento}

Este aspecto refere-se ao início da mastigação, correspondendo à fase incisal da mesma, cabendo ao examinador observar o local da cavidade oral onde a incisão ocorre ${ }^{11}$. Pode localizar-se na região anterior, quando realizada com os dentes incisivos centrais e laterais; na região lateral, quando realizada pelos incisivos laterais em conjunto com os caninos e até pré-molares ou, ainda, região posterior, quando realizada com os pré-molares e molares. Pode-se, também, observar que o paciente posiciona o alimento entre os dentes, mas não realiza a atividade de corte, utilizando os dentes anteriores como alavanca para quebrar o alimento. Além disso, o paciente pode partir o alimento com as mãos antes de posicioná-lo entre os dentes, não ocorrendo, portanto, a fase incisal da mastigação ${ }^{6,7}$. Em condições favoráveis, em geral, esta preensão do alimento é realizada na região anterior.

\section{Movimento mandibular}

Deve ser avaliado por meio de palpação, percebendo-se a excursão da mandíbula, somado à observação visual. Considera-se adequado os movimentos de abertura inclinados para o lado de não trabalho e os de fechamento dirigidos para o lado de trabalho, isto é, o lado da trituração ${ }^{12}$, portanto, presença de movimentos laterais. Quando 0 alimento encontra-se mais triturado, o movimento predominante da mandíbula se dá no plano vertical ${ }^{13}$.

\section{Lado inicial da mastigação}

Deve-se observar os movimentos mandibulares no primeiro ciclo mastigatório, em que a mandíbula deve estar lateralizada para o lado inicial e os dentes deste mesmo lado em oclusão. Também pode ser utilizada a palpação, com um leve toque nos músculos masseteres, percebendo qual o lado de maior contração do músculo. Além disso, pode-se observar o $1^{\circ}$, $3^{\circ}$, $5^{\circ}$ e $7^{\circ}$ ciclos mastigatórios, repetindo-se o teste 4 vezes. Se durante três testes o lado inicial da mastigação coincidir, este é o lado sugestivo de preferência mastigatória ${ }^{14}$.

\section{Tipo mastigatório}

Verifica-se todos os ciclos mastigatórios, identificando o número de ciclos realizados em cada lado (direito e esquerdo). Recomenda-se que este procedimento seja realizado posteriormente, a partir da análise da filmagem.

Segundo critérios utilizados por alguns autores ${ }^{7,15}$, a mastigação será classificada como unilateral predominante quando houver mais de $66 \%$ dos ciclos mastigatórios realizados em um único lado. Quando houver mais de 95\% de ciclos mastigatórios realizados em um único lado, será classificada como unilateral crônica. Além destas classificações, o tipo mastigatório pode ser bilateral alternado, havendo nesses casos, predominância de movimentos verticais e laterais da mandíbula ou bilateral simultâneo, quando estes movimentos forem verticais ${ }^{16}$.

\section{Musculatura perioral}

Por meio da observação visual verifica-se a participação da musculatura perioral durante os ciclos mastigatórios. Para a realização da mastigação, há participação da musculatura perioral, que deve ser discreta. No entanto, quando a participação dessa musculatura for acentuada ou quase estática, há indícios de anormalidade ${ }^{17}$.

\section{Lábios}

Deve ser observada a postura labial durante a realização da função mastigatória, verificando se os mesmos permanecem selados, pois evitam o escape anterior do alimento, bem como auxiliam na manutenção da pressão intra-oral para a ejeção do bolo alimentar para a faringe ${ }^{18,19}$. Também, os lábios podem encontrar-se afastados durante toda a mastigação, sendo visualizado o bolo alimentar dentro da cavidade oral ou, ainda, encontrarem-se ora selados e ora afastados ${ }^{16}$.

\section{Tremor}

Avaliado por meio de observação dos tecidos periorais durante a realização da função mastigatória, sendo necessário especificar em qual estrutura oral o mesmo ocorre. A presença deste sinal sugere incoordenação dos movimentos mandibula- 
res, hipotonicidade dos músculos mastigatórios e, principalmente alterações neurológicas ${ }^{20}$.

\section{Contração de Masseteres}

Avaliada por meio de palpação digital, posicionase os dedos bilateralmente na face do paciente na região dos músculos, enquanto o mesmo executa a função mastigatória. Com um leve toque nessa região, percebe-se sua contração, classificando-a, subjetivamente, em forte, regular ou fraca. A contração do masseter durante a função mastigatória pode estar intimamente relacionada com o tônus deste músculo ${ }^{6,7,16}$.

Esta contração muscular também pode ser verificada por meio de eletromiografia, uma vez que os fonoaudiólogos entrevistados relataram sua utilização na avaliação clínica. Vale enfatizar que, o registro do potencial de ação é variado conforme a característica do alimento, quantidade do mesmo, tipologia facial, entre outros fatores ${ }^{8,21-23}$. Cabe lembrar, também, que o registro eletromiográfico varia de um equipamento para outro, o que dificulta a comparação dos mesmos entre os diferentes equipamentos.

\section{Coordenação dos movimentos}

A partir da visualização e análise da filmagem, observam-se os movimentos mandibulares de fechamento, lateralização e abertura, assim como os movimentos dos lábios e da língua quanto à pre- cisão dos mesmos. A presença de incoordenação dos movimentos mandibulares sugere alterações no controle motor dos músculos mastigatórios ${ }^{13,17}$.

\section{Escape anterior do alimento}

Por meio de visualização, verifica-se a presença de acúmulo de alimento nas comissuras labiais, bem como o escoamento do mesmo entre os lábios ${ }^{24}$.

\section{Amassamento com a língua}

A partir da visualização e análise da filmagem, verifica-se a presença de esmagamento do bolo alimentar com a língua contra o palato duro, notandose a movimentação vertical ou póstero-anterior da língua. Quando essa situação ocorre, em geral, os lábios encontram-se afastados. Entretanto, se os lábios estiverem selados, observa-se movimentação mandibular vertical e anterior ${ }^{25}$.

\section{Movimento de cabeça}

Observa-se durante a avaliação direta e na análise da filmagem, se há compensação postural da cabeça durante a mastigação. Por esta razão, recomenda-se que o paciente não esteja com a cabeça apoiada, de forma a não limitar os movimentos espontâneos apresentados. Utiliza-se como referência, o painel com os planos horizontal e vertical, localizados atrás do paciente, como mostra a Figura 2. Pode-se encontrar a cabeça em posição centralizada, mantendo o plano oclusal paralelo ao

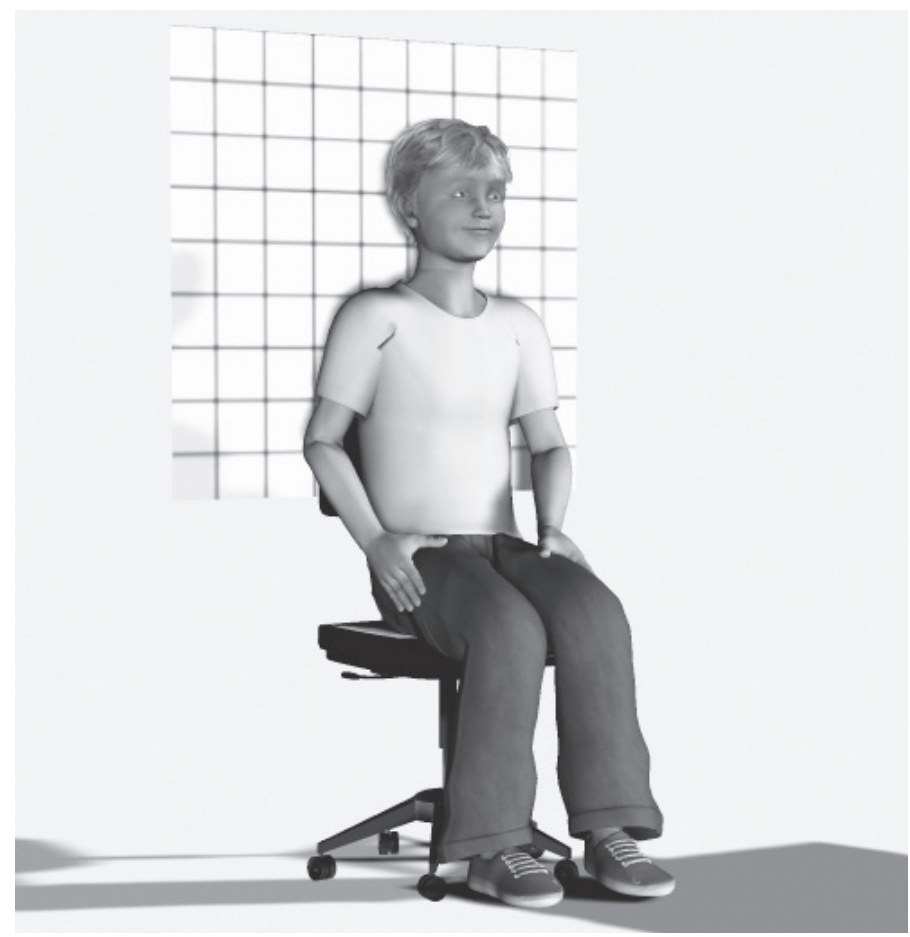

Figura 2 - Posicionamento ideal de um paciente a ser avaliado quanto à função mastigatória 
solo com a cabeça estática. Ainda pode ocorrer a inclinação da cabeça para um dos lados, ou ainda, realizar movimentos diversos como se estivesse "ajudando" os dentes a realizarem trituração ${ }^{26,27}$.

\section{Local da trituração do alimento}

A partir da visualização e análise da filmagem, nota-se o local da cavidade oral em que está ocorrendo a trituração do alimento. Esta pode localizar-se na região posterior (molares e pré molares), mas também pode ocorrer na região anterior, com os dentes anteriores, como compensação, principalmente nos casos de má-oclusão ${ }^{6,28}$. Quando os lábios encontram-se afastados, esta observação é mais fácil, pois se verifica a localização anterior do alimento. Entretanto, quando os lábios estiverem selados, verifica-se a partir da movimentação mandibular vertical e protrusiva ${ }^{29}$.

\section{Respiração durante a mastigação}

Durante o exame direto ou na análise da filmagem, verifica-se o modo respiratório durante a função mastigatória, observando-se a coordenação entre essas duas funções. Se os lábios estiverem selados durante a mastigação, a respiração está ocorrendo por via nasal. Porém, se os lábios estiverem afastados assistematicamente ou abertos, apresentando respiração ruidosa e ofegante, trituração ineficiente dos alimentos, além da diminuição do número de ciclos mastigatórios e do tempo mastigatório, tais características são sugestivas de respiração oronasal ou oral ${ }^{2,30}$. Uma vez que exista indicativo de respiração oronasal ou oral, deve-se encaminhar o paciente para um médico otorrinolaringologista para investigação da permeabilidade das vias aéreas superiores.

\section{Ruídos nas ATMs}

Cabe lembrar que uma articulação saudável não apresenta ruídos. Assim, deve ser investigado, por meio da ausculta das ATMs com estetoscópio, a presença desse evento, conforme já relatado na literatura ${ }^{31}$. Quando se nota algum ruído nesta articulação, os mesmos devem ser classificados em estalidos ou crepitação, verificando se os mesmos são unilaterais ou bilaterais. Estalo é caracterizado por um ruído derivado do movimento do côndilo ao passar pela banda posterior do disco articular; pode ocorrer na abertura ou no fechamento da boca, representando algum grau de deslocamento do disco articular. Enquanto que a crepitação, referida como um "ruído de areia", "pisar em folhas secas" ou "ranger de ossos fraturados", ocorre na presença de deslocamento ou de lesões do disco articular e de doença degenerativa articular, uma vez que a superfície articular torna-se irregular ${ }^{6,31}$.

\section{Tempo mastigatório}

O tempo mastigatório, em segundos, será mensurado utilizando-se um cronômetro, o qual será acionado imediatamente no início da fase incisal e interrompido quando iniciar a primeira deglutição, observada pela elevação da laringe, característica do início da fase faríngea da deglutição, como manobra para proteção das vias aéreas ${ }^{24}$. Este procedimento deve ser repetido três vezes para se certificar do tempo mastigatório. Estudos mostram que o tempo médio está entre $11,17 \pm 1,78^{8}$ e $15,4 \pm 3,6$ segundos para o biscoito tipo waffer (porção de 2 $\mathrm{cm})^{9}$ e de $26,3 \pm 8,8$ segundos para $1 / 4$ do mini pão francês ${ }^{9}$ ou de $12,79 \pm 2,6$ segundos uma porção de pão francês, equivalente a $1 / 4$ de uma fatia de $1 \mathrm{~cm}$ de espessura ${ }^{8}$.

\section{Número de ciclos mastigatórios}

Deve ser analisado por meio da filmagem, observando-se a quantidade de movimentos mastigatórios realizados durante a mastigação de um determinado alimento. Na literatura, há relato de $14^{8}$ a 15 ciclos mastigatórios ${ }^{21}$, em média, para o pão francês, e de 13 ciclos para o biscoito tipo waffer ${ }^{8}$.

\section{Formação do bolo}

Em um segundo teste, o avaliador deve orientar o paciente a não deglutir o alimento triturado, já nos momentos finais da mastigação que antecedem a deglutição. O mesmo deve deixar o bolo alimentar pronto para ser deglutido, posicionado de maneira habitual e, em seguida, mostrá-lo ao avaliador antes de degluti-lo. $\mathrm{O}$ avaliador observa a formação do bolo alimentar, se o mesmo é coeso, bem como o local onde o mesmo foi posicionado, se na região do sulco longitudinal da língua ou espalhado pela cavidade oral. $O$ bolo alimentar pode não ter sido formado totalmente, mas com presença de resíduos na cavidade, ou ainda, permanecendo o alimento espalhado pelo interior da cavidade oral sem formação do bolo alimentar. $O$ adequado é que todo o alimento esteja posicionado sobre a língua, na região central, não restando resíduos espalhados no interior da cavidade oral ${ }^{16}$.

Quando o paciente abrir a boca para o avaliador observar o bolo alimentar, este irá verificar se o alimento foi devidamente triturado, apresentando uma forma homogênea, ou se restam partículas grandes que não foram trituradas adequadamente, mas que serão deglutidas desta forma ${ }^{6,32}$.

Deve-se lembrar que tanto o número de ciclos mastigatórios, quanto a formação do bolo alimentar podem variar de acordo com as características e tamanho do alimento testado, como já foi descrito nos itens 1 e 2. 


\section{Presença de tosse, engasgo, dispnéia ou modificação na coloração facial}

Estes aspectos devem ser observados durante a mastigação, o esperado é que o paciente consiga conter o bolo na cavidade oral até o momento da deglutição. Todos estes sinais podem ser indicativos de escape posterior do alimento durante a mastigação ${ }^{24}$.

\section{Dor durante a mastigação}

Deve-se verificar durante a mastigação quaisquer modificações na expressão facial do paciente, bem como questioná-lo sobre a presença de dor ou desconforto em algum momento da realização desta função, em que local e em que intensidade ocorreu ${ }^{6,7}$.

\section{Teste de eficiência mastigatória}

A verificação da trituração adequada do bolo alimentar pode ser realizada a partir do teste de eficiência mastigatória. Recomenda-se o uso do método de utilização de amêndoas como alimento de teste e um sistema de tamises com peneiras com orifícios variados, como ilustrado na Figura 3 ${ }^{10}$. Solicita-se ao indivíduo a ser avaliado, a mastigação de 5 amêndoas. A $1^{\underline{a}}$ amêndoa deve ser mastigada de forma habitual, a $2^{\underline{a}}$ amêndoa deve ser triturada por 10 segundos, a $3^{\underline{a}}$ e a $4^{\mathrm{a}}$ amêndoas por 20 e 40 segundos respectivamente e, a $5^{a}$ amêndoa utiliza-se o mesmo tempo e número de ciclos mastigatórios realizados durante a mastigação da $1^{\underline{a}}$ amêndoa.
O sistema de tamises, composto por três peneiras com orifícios dos respectivos tamanhos: 4,75 $\mathrm{mm}, 4 \mathrm{~mm}$ e $2 \mathrm{~mm}$, dispostas de maneira que os alimentos fragmentados passem progressivamente do maior para o menor orifício, após lavadas por água corrente por 60 segundos. As partículas de cada amêndoa são então classificadas de acordo com seus tamanhos: A, com tamanho acima de $4,75 \mathrm{~mm}$; B com tamanho menor que $4,75 \mathrm{~mm}$ e maior que $4 \mathrm{~mm}$ e $C$ com tamanho menor que $4 \mathrm{~mm}$ e maior que $2 \mathrm{~mm}$. A partir dessa análise, a eficiência mastigatória é classificada em ótima, quando houver ausência de partículas A e presença de poucas partículas $B$ após a fragmentação da $2^{\text {a }}$ amêndoa; boa, quando houver ausência de partículas $A$ e presença de poucas partículas $B$ após a fragmentação da $3^{a}$ amêndoa; regular, quando tiver poucas partículas $A$ e $B$ após a fragmentação da $3^{\text {a }}$ amêndoa; ruim, quando o paciente não se encaixa em nenhuma das classificações anteriores, porém não há partículas $A$ após a fragmentação da $4^{a}$ amêndoa e péssima, quando o paciente não se encaixa em nenhuma das classificações anteriores, porém há presença da partículas $A$ após a fragmentação da 4ª amêndoa.

\section{DISCUSSÃO}

A Fonoaudiologia tem buscado objetividade nas avaliações que comprovem a efetividade da intervenção fonoaudiológica, incentivando os pesquisadores a investigarem as variações da norma-

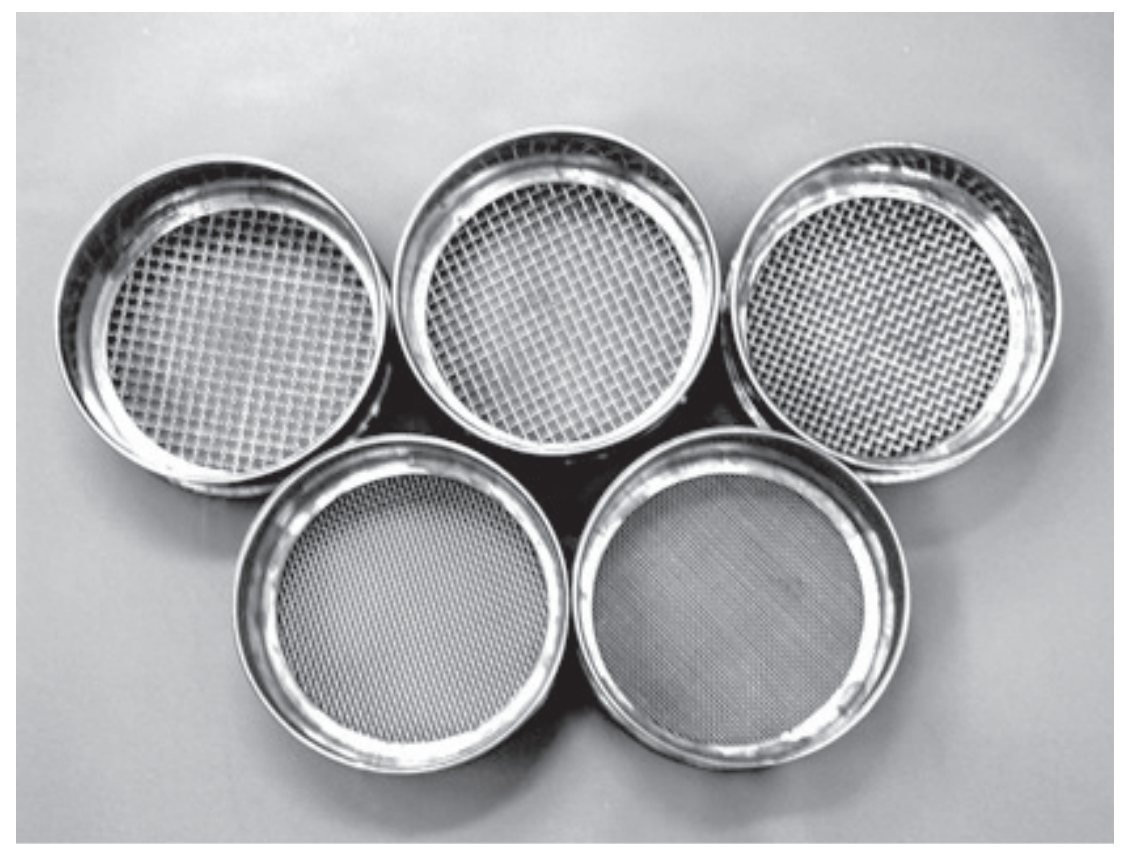

Figura 3 - Sistema de tamises, peneiras de diferentes tamanhos de orifícios 
lidade para que, somente com estes dados, possa aplicá-los na prática clínica. A função mastigatória desperta interesse de várias ciências como a Fonoaudiologia, Odontologia, Fisioterapia e Medicina. Sabendo, assim, que o fonoaudiólogo é o profissional apto para prevenir, diagnosticar e reabilitar as alterações das funções estomatognáticas, a mastigação torna-se parte importante da atuação deste profissional.

A ideia de se propor uma avaliação clínica da função mastigatória baseada em estudos já realizados, de diversas áreas, não bastaria, se esta avaliação não fosse, além de completa e com respaldo científico, acessível para os fonoaudiólogos aplicarem em sua prática clínica, nos seus diferentes locais de atuação.

Uma das primeiras preocupações com a avaliação da função mastigatória deve ser em relação ao tipo e à quantidade do alimento a ser oferecido ao paciente durante a avaliação clínica, para que se tenham parâmetros normativos e de comparação entre os diferentes casos. Sabe-se que o tipo de alimento modifica a função mastigatória, em relação ao tempo dos ciclos e ato mastigatório, número, frequência e ritmo dos ciclos mastigatórios e movimentos mandibulares ${ }^{23,32-34}$.

Assim, ao mastigar um alimento mais consistente e numa maior quantidade, haverá o aumento do número de ciclos mastigatórios, bem como da amplitude dos movimentos mandibulares, o que demonstra a necessidade de se utilizar, sempre que possível, a mesma quantidade de alimento na avaliação, para que se tenham parâmetros de comparação.

São necessários que os dados da história clínica sejam delineadores da escolha do alimento, de acordo com a queixa, preferência alimentar e dificuldades apresentadas pelo paciente, isso porque existe o mecanismo modulador da força mastigatória por meio dos receptores periodontais, que modifica a força mastigatória e o número de ciclos mastigatórios, de acordo com as características físicas do alimento ${ }^{29}$. Isto quer dizer que, de nada adiantaria, para fins de comparação, a utilização de um mesmo alimento nas avaliações se, a quantidade deste não estiver padronizada.

Encontra-se, na literatura, um estudo sobre a avaliação do número de ciclos mastigatórios em indivíduo normais e com disfunção craniomandibular (DCM), em alimentos de diferentes consistências ${ }^{21}$. Os valores de referência encontrados, no grupo controle estudado, foram, em média, de 12 tanto para $1 / 4$ de fatia de pão francês, quanto para $1 \mathrm{~cm}$ de maça cortada em forma de bolinhas e 11 ciclos para fatias de $1 \mathrm{~cm}$ de banana e uma semente de castanha. Já de acordo com outro estudo ${ }^{8}$, o número de ciclos mastigatórios para o biscoito tipo waffer $(2 \mathrm{~cm})$ e o pão francês $(2 \mathrm{~cm})$ foi de 14,85 e de 14,78 , respectivamente.

Assim, sugere-se que a avaliação da mastigação seja realizada com pão ou biscoito tipo waffer, sendo o primeiro de custo baixo e fácil aceitação entre os pacientes ${ }^{16} \mathrm{e}$ o segundo com um tempo maior de conservação, sem modificação de suas propriedades.

Já o tempo de mastigação foi avaliado ${ }^{21} \mathrm{em}$ alimentos com diferentes consistências: 1 pedaço de maçã com casca $(1 \mathrm{~cm}$ de diâmetro, cortada em forma de bolinhas), 1 rodela de banana $(1 \mathrm{~cm}$ de espessura), 1/4 do mini pão francês, 1 castanha de caju e encontrou os seguintes valores, para indivíduos normais: $74 \mathrm{~s}, 75 \mathrm{~s}, 77 \mathrm{~s}$ e $77 \mathrm{~s}$, respectivamente. Em outro estudo ${ }^{9}$ que também avaliou a duração dos ciclos mastigatórios nos mesmos alimentos e nas mesmas quantidades, além de avaliar 1 bolacha tipo waffer e 1/4 de biscoito tipo água e sal, foram obtidos os seguintes tempos de mastigação, em segundos: Banana: 9,6; Pão: 26,3; Waffer. 15,4; Bolacha de água e sal: 11,4; Maçã: 10,4 e Castanha: 11,6. A diferença nos tempos de mastigação destes alimentos testados corroboram os dados da literatura, em relação à variação das características da mastigação de acordo com cada tipo de alimento.

$\mathrm{Na}$ literatura há relato de correlação entre a duração da mastigação obtida na avaliação com o cronômetro e a duração da mastigação obtida na avaliação eletromiográfica (EMG), observando que, a média da duração do tempo mastigatório para o biscoito waffer foi de 12,95 e para o pão foi de 15,39 , havendo uma correlação positiva entre a duração do tempo total da mastigação da avaliação EMG e da avaliação com o cronômetro ${ }^{8}$.

Se o pão ou o biscoito waffer forem os alimentos escolhidos para a realização da avaliação, na quantidade proposta, os tempos de mastigação devem estar de acordo com a literatura ${ }^{8,9} \mathrm{e}$, sendo que um tempo maior corresponderia a uma mastigação lenta e um tempo menor, a uma mastigação rápida, devendo-se levar em consideração a quantidade oferecida para teste. Estes dados devem ser relacionados com os dados colhidos na história clínica, na qual o paciente deverá comparar sua velocidade de mastigação com os outros membros da família ou pessoas com quem divida o momento de alimentação.

Após a definição dos tipos e das quantidades dos alimentos a serem utilizados, deve-se solicitar ao paciente que mastigue de forma habitual e seja o quanto menos interferido pelo avaliador, de modo que a mastigação não se modifique na presença do mesmo ${ }^{16}$. 
Deve ser observada, inicialmente, a forma de corte do alimento, também chamada de preensão do alimento ou mordida, correspondente à fase incisal da mastigação. A forma de preensão do alimento nos fornece dados importantes a respeito da condição dentária do indivíduo, força de mordida e condição dos movimentos mandibulares. Fatores como deformidades dentofaciais, anquilose de ATM e perdas de elementos dentários anteriores podem alterar este aspecto ${ }^{6,7,31}$. Diante de boa condição dentária e oclusal, além da adequada movimentação da mandíbula, espera-se que esta preensão ocorra na região anterior ${ }^{2,16,31}$, dado o papel cortante dos dentes incisivos. Entretanto, diante de alterações estruturais, esta pode ocorrer com os dentes laterais ou, ainda realizar o corte com as mãos, como forma adaptativa do sistema estomatognático ${ }^{2,6,7}$.

Os movimentos mandibulares devem ser observados em relação à coordenação e a precisão dos movimentos e a presença de tremor. E, apesar de haver ampla variação dos movimentos mandibulares que representam padrões variados de mastigação, dentro da normalidade, segundo análises computadorizadas destes movimentos, é possível a observação dos movimentos mandibulares por meio da movimentação da musculatura perioral ${ }^{17}$. Com isso, a participação da musculatura perioral deve ser correspondente aos movimentos mandibulares; uma participação exagerada ou quase estática destes tecidos indica anormalidade de movimentos.

Consideram-se adequados os movimentos de abertura inclinados para o lado de não trabalho e os de fechamento dirigidos para o lado de trabalho, isto é, o lado da trituração ${ }^{12}$. Quando o alimento encontra-se mais triturado, o movimento predominante da mandíbula se dá no plano vertical ${ }^{13}$. Desta forma, pode-se classificar os movimentos mandibulares em laterais, quando há lateralização do alimento na cavidade oral e alternância nos lados de trabalho ou, ainda, verticais quando os dois lados forem de trabalho ${ }^{31,35}$. Os movimentos mandibulares se alteram de acordo com o grau de trituração do alimento, onde quanto mais triturado, maior predomínio de movimento vertical da mandíbula ${ }^{23}$.

Assim, no processo de mastigação normal, os fenômenos espaciais que ocorrem na mandíbula durante os ciclos mastigatórios são as fases de abertura da boca, na qual a mandíbula se abaixa, com relaxamento dos músculos levantadores e a contração isotônica dos músculos abaixadores da mandíbula; de fechamento da boca, momento no qual a mandíbula se eleva pela contração isotônica dos músculos levantadores e pelo relaxamento reflexo dos músculos abaixadores da mandíbula; e fase oclusal, na qual há contato e intercuspida- ção dos dentes, gerando forças inter-oclusais em decorrência da contração isométrica dos músculos levantadores da mandíbula, que partem o alimento em partículas menores.

Os movimentos mandibulares poderão estar limitados na presença de DTM anquilose manbibular $6,7,31$, bem como devido à presença de próteses sem retenção adequada ${ }^{10}$. Já a incoordenação, imprecisão e presença de tremor durante a execução destes movimentos podem ocorrer em alterações neurológicas degenerativas como, na esclerose lateral amiotrófica e doença de Parkinson ${ }^{24}$.

Tosse, engasgos, dispnéia e modificações na coloração do paciente podem ocorrer durante a mastigação, devido ao escape posterior do alimento, cujo avaliador também deve atentar-se, principalmente, na presença de alterações neurológicas. Geralmente, ocorre por dificuldade de manter o bolo alimentar na cavidade oral, deixando-o escoar para a faringe antes do disparo do reflexo de deglutição. Esta dificuldade pode ser decorrente de alterações sensitivas ou motoras no sistema digestivo ${ }^{2,24}$.

Muitas vezes o paciente realiza compensações da função, apresentando movimentos de cabeça, trituração do alimento com os dentes anteriores, amassamento do alimento com a língua que, também devem ser considerados durante a avaliação, para análise das causas da disfunção ${ }^{24}$.

Durante os movimentos mandibulares é necessário analisar a postura dos lábios, que devem estar selados, a fim de evitar o escape anterior dos alimentos, bem como auxiliam na manutenção da pressão intra-oral para a ejeção do alimento para a faringe para que ocorra o processo normal de deglutição ${ }^{2}$. Lábios desocluídos durante a mastigação podem estar diretamente relacionados com a presença de deformidades dentofaciais, como dimensão vertical do terço inferior da face aumentada e, principalmente, alteração do modo respiratório ${ }^{18,19}$.

De acordo com os questionários respondidos, houve a sugestão de que fosse observado o lado em que se inicia a mastigação. Isto pode ser realizado pela observação dos movimentos mandibulares, onde a mandíbula deve estar lateralizada para aquele lado e os dentes deste lado em oclusão, ou por meio de palpação tocando levemente a região dos músculos masseteres, percebendo em qual lado há maior contração. Em seguida, utiliza-se a mesma estratégia para classificar a contração do músculo masseter. De acordo com a literatura ${ }^{14}$, o lado em que se inicia a função mastigatória, geralmente é coincidente com o tipo mastigatório. Tal investigação complementa a avaliação do tipo mastigatório. 
Assim, o tipo de mastigação, que diz respeito ao lado preferencial de mastigação, pode ser concordante com o lado em que se inicia a mastigação ou com a lateralidade cerebral ${ }^{14}$ ou ainda, segundo o critério utilizado por alguns autores ${ }^{7,15}$, é considerada uma predominância unilateral, quando houver mais de $66 \%$ dos movimentos mastigatórios daquele lado e, mastigação unilateral crônica, quando houver mais de $95 \%$ de movimentos daquele lado.

Sugere-se a proposta deste autor ${ }^{15}$, classificando o tipo mastigatório de bilateral alternado, bilateral simultâneo, unilateral crônico ou predominante unilateral, por ser um método mais fácil de ser aplicado e relatado em pesquisas científicas. Para a classificação do tipo mastigatório, é necessária a análise do número de ciclos mastigatórios totais e de cada lado. Aconselha-se que esta análise seja realizada posteriormente, a partir da filmagem.

A presença de ruídos nas ATMs podem indicar DCM, assim devem ser investigados por meio da ausculta da ATM ${ }^{31}$. Casos de DCM têm sido amplamente estudados, uma vez que possuem inúmeros fatores desencadeantes, tornando muitos indivíduos expostos a tal disfunção ${ }^{6,7}$.

Para se investigar a formação e a trituração do bolo, deve-se orientar o paciente a não deglutir o alimento triturado e sim, prepará-lo para a deglutição, devendo ser mostrado antes da deglutição, para verificar a fase de organização. Deve ser observado se há formação de bolo, isto é se aquele alimento triturado está centralizado na língua e se, além de centralizado está bem triturado, se apresenta uma forma homogênea ou ainda são visualizadas partículas não trituradas. A trituração inadequada do bolo alimentar diminui a superfície de contato para a ação das enzimas digestivas, o que dificulta a degradação do alimento ingerido ${ }^{29}$.

O período entre a fase incisal e o momento que antecede a deglutição com a elevação da laringe, corresponde ao tempo mastigatório, mensurado por meio de cronômetro. Sabe-se que o tempo de mastigação varia de acordo com o tipo de alimento, por isso é necessário padronizá-lo ${ }^{29}$.
O questionamento sobre a presença de dor ou desconforto durante a mastigação, em que intensidade, em que local e em que momento aconteceu deve fazer parte da avaliação, para que sejam realizados o diagnóstico preciso e o direcionamento adequado de tratamento ${ }^{6}$.

Foi observado que quanto mais lenta é a mastigação, melhor é a trituração do bolo, sendo este melhor formado. Em contrapartida, a mastigação rápida não promove uma boa trituração do alimento e mantém as partículas espalhadas na boca, sem a formação adequada do bolo, o que dificulta a deglutição e a digestão dos alimentos. O método proposto na literatura ${ }^{10}$ apresenta etapas claras e bem definidas, além de ser prática e viável de ser realizado na prática clínica, para a avaliação da trituração do bolo alimentar, isto é, da eficiência mastigatória.

A classificação da eficiência em ótima, boa, regular, ruim e péssima fornece dados de como se encontra a função mastigatória de acordo com as características observadas em cada aspecto, uma vez que a eficiência mastigatória é o resultado final da mastigação como um todo.

Ao final deste estudo verifica-se que apesar da investigação da literatura, é importante enfatizar que para a avaliação clínica, ainda existem poucas normalizações, pois muitos aspectos investigados ainda baseiam-se em critérios subjetivos, os quais dependem da experiência do profissional, inviabilizando, muitas vezes, comparações dos resultados entre diferentes profissionais e centros de pesquisa. Este fato evidencia a necessidade de novos estudos nessa área.

\section{CONCLUSÃO}

Este trabalho propôs apresentar um roteiro para a avaliação clínica da mastigação e desta forma, acredita-se ter contemplado aspectos importantes da mastigação, proporcionando ao fonoaudiólogo uma melhor definição de critérios a serem utilizados durante a avaliação da função mastigatória. 


\section{ABSTRACT}

Purpose: to elaborate a protocol for the clinical evaluation of the masticatory function, aiming at aiding the speech pathologist to identify and interpret the clinical signals of the masticatory disorders, in its dynamics, in addition to allowing for inter-judge comparison and treatment continuation. Methods: this protocol was elaborated based on the clinical practice of the speech pathologists that answered to a questionnaire sent by email; in the literature, according to the identification of the common points to all chewing evaluation protocols; in addition to the experience of the speech pathologists of HRAC/USP. Results: the protocol for the clinical chewing evaluation considers aspects related to morphologic aspects and neurophysiology of the masticatory function. Conclusion: this study contributes to Speech Pathology, once that it searches, in the most possible complete manner, criteria for evaluating the chewing, guiding the clinical speech-language performance based on evidences.

KEYWORDS: Evaluation, Mastication; Etomatognatic System

\section{REFERÊNCIAS}

1. Comitê de Motricidade Oral. Sociedade Brasileira de Fonoaudiologia. Documento oficial 04/2007.

2. Cattoni DM. Alterações da mastigação e deglutição. In: Ferreira LP, Befi-Lopes DM, Limongi SCO, organizador. Tratado de fonoaudiologia. São Paulo: Roca; 2004. p.277-91.

3. Felício CM. Desenvolvimento normal das funções estomatognáticas. In: Ferreira LP, BefiLopes DM; Limongi SCO, organizador. Tratado de Fonoaudiologia. São Paulo: Roca; 2004. p.195-211.

4. Zhao L, Monahan R. Functional assessment of the stomatognathic system. Clin Plast Surg. 2007; 34(3):1-9.

5. Gomes FCS, Melo LF, Chiappetta ALML. Aspectos do padrão mastigatório na dentição decídua e mista em crianças de três a nove anos. Rev CEFAC. 2006; 8(3):313-9.

6. Felício CM, Melchior MO, Silva MAMR, Celeghini RMS. Desempenho mastigatório em adultos relacionado com a desordem temporomandibular e com a oclusão. Pró-Fono. 2007; 19(2):151-8.

7. Felício CM, Mazzetto MO, Bataglion C, Silva MAMR, Hotta TH. Desordem temporomandibular: análise da frequência e severidade dos sinais e sintomas antes e após a placa de oclusão. J Bras Ortodon Ortop Facial. 2003; 8(43):48-57.

8. Arrais RD, Genaro KF, Sampaio ACM. Função mastigatória em indivíduos normais: duração do ato e do ciclo mastigatório. Anais do $12^{\circ}$ Simpósio internacional de Iniciação Científica da Universidade de São Paulo; 2004, 23-24 nov; Ribeirão Preto, Brasil. p. 788.

9. Melo TM, Arrais RD, Genaro KF. Duração da mastigação de alimentos com diferentes consistências. Rev Soc Bras Fonoaudiol. 2006; 11(3):170-4.

10. Carvalho LEP. Avaliação do nível de satisfação, capacidade, eficiência e performance mastigatórias em pacientes reabilitados com próteses fixas totais inferiores sobre implantes, sob carga imediata [dissertação]. Bauru (SP): Universidade do Sagrado Coração; 2002.

11. Berretin-Félix G, Jorge TM, Genaro KF. Intervenção fonoaudiológica em pacientes submetido à cirurgia ortognática. In: Ferreira LP, Befi-Lopes DM, Limongi SCO, organizador. Tratado de fonoaudiologia. São Paulo: Roca; 2004. p. 495-511.

12. Mioche L, Bourdiol P, Monier S. Chewing behavior and bolus formation during mastication of meat with different textures. Arch Oral Biol. 2003; 48(3):193-200.

13. Komiyama $O$, Asano $T$, Suzuki $H$, Kawara $M$, Wada M, Kobayashi K, et al. Mandibular condyle movement during mastication of foods. J Oral Rehabil. 2003; 30(6):592-600.

14. Nissan J, Gross MD, Shifman A, Tzadok L, Assif D. Chewing side preference as a type of hemispheric laterality. J Oral Rehabil. 2004; 31(5):412-6.

15. Tay DK. Physiognomy in the classification of individuals with a lateral preference in mastication. $J$ Orofac Pain. 1994; 8(1):61-72.

16. Felício CM, Ferreira CLP. Protocol of orofacial myofunctional evaluation with scores. Int $\mathrm{J}$ Pediatr Otorhinolaryngol. 2008; 72(3):367-75.

17. Pignataro Neto G, Bérzin F, Rontani RMP. Identificação do lado de preferência mastigatória através de exame eletromiográfico comparado ao visual. Rev Dent Press Ortodon Ortop Facial. 2004; 9(4):77-85.

18. Cunha DA, Silva GAP, Motta MEFA, Lima $\mathrm{CR}$, Silva HJ. A respiração oral em crianças e 
suas repercussões no estado nutricional. Rev CEFAC. 2007; 9(1):47-54. dx.doi.org/10.1590/ S1516-18462007000100007

19. Duarte LIM, Ferreira LP. Respiração e mastigação: estudo comparativo. Rev Dental Press Ortodon Ortop Facial. 2003; 8(4):79-87.

20. Groher ME. Distúrbios de deglutição em idosos. In: Furkim AM, Santini CS, organizador. Disfagias orofaríngeas. Carapicuiba: Pró-Fono; 1999. p.97-108.

21. Berretin-Felix G, Genaro KF, Trindade IEK, Trindade Júnior AS. Masticatory function in temporomandibular dysfunction patients: eletromyographic evaluation. J Appl Oral Sci. 2005; 13(4):360-5.

22. Oncins MC, Freire RMAC, Marchesan IQ. Mastigação: análise pela eletromiografia e eletrognatografia: seu uso na clínica fonoaudiológica. Dist Comun. 2006; 18(2):155-65.

23. Kohyama K, Mioche L, Bourdiol P. Influence of age and dental status on chewing behaviour studied by EMG recordings during consumption of various food samples. Gerodontol. 2003; 20(1):15-23.

24. Padovani AR, Moraes DP, Mangili LD, Andrade CRF. Protocolo fonoaudiológico de avaliação do risco para disfagia (PARD). Rev Soc Bras Fonoaudiol. 2007; 12(3):199-205.

25. Nishigawa K, Nakano M, Bando E. Study of jaw movement and masticatory muscle activity during unilateral chewing with and without balancing side molar contacts. J Oral Rehabil. 1997; 24(9):691-6.

26. Ueda $H M$, Kato $M$, Saifuddin $M$, Tabe $H$, Yamaguchi K, Tanne K. Differences in the fatigue of masticatory and neck muscles between male and female. J Oral Rehabil. 2002; 29(6):575-82.
27. Cesar AM, Garibaldi A, Di Ninno CQM, Krakauer L. A influência da postura anteriorizada da cabeça no padrão mastigatório. Rev CEFAC. 2004; 6(3):259-65.

28. PastanaSG, CostaSM, Chiappetta ALML. Análise da mastigação em indivíduos que apresentam mordida cruzada unilateral na faixa-etária de 07 a 12 anos. Rev CEFAC. 2007; 9(3):339-50. dx.doi. org/10.1590/S1516-18462007000300008

29. Douglas CR. Tratado de fisiologia aplicada à fonoaudiologia. 1. ed. São Paulo: Robe; 2002.

30. Silva MAA, Natalini V, Ramires RR, Ferreira LP. Análise comparativa da mastigação de crianças respiradoras nasais e orais com dentição decídua Rev CEFAC. 2007; 9(2):190-8. dx.doi.org/10.1590/ S1516-18462007000200007

31. Bianchini EMG. Articulação temporomandibular: implicações, limitações e possibilidades fonoaudiológicas. 1. ed. Carapicuiba: Pró-Fono; 2000. 402p. 32. Hatch JP, Shinkai RS, Sakai S, Rugh JD, Paunovich ED. Determinants of masticatory performance in dentate adults. Arch Oral Biol. 2001; 46(7):641-8.

33. Felício CM, Fernandes RSM, Silva MAMR. Investigação da relação entre o tipo mastigatório e o limite de movimento lateral da mandíbula. Rev Soc Bras Fonoaudiol. 2005; 10(2):71-6.

34. Filipic S, Keros J. Dynamic influence of food consistency on the masticatory motion. J Oral Rehabil. 2002; 29(5):492-6.

35. Teixeira ML. Avaliação do movimento mandibular em pacientes com fissura transforame incisivo. [dissertação]. Bauru (SP): Universidade de São Paulo; 2000.

DOI: 10.1590/S1516-18462009005000030

RECEBIDO EM: 10/04/2008

ACEITO EM: 19/01/2009

Endereço para correspondência:

Melina Evangelista Whitaker

Rua Dr. Antônio Prudente, 4-152

Bauru - SP

CEP: 17016-010

E-mail: mirian3m@uol.com.br 